\title{
RDUS
}

Revue de DROIT

UNIVERSITÉ DE SHERBROOKE

Titre : L'ACCÈS AUX SERVICES D'AIDE À L'HYGIÈNE EN RÉSIDENCE POUR PERSONNES ÂGÉES : UNE ATTEINTE AUX PRINCIPES D'ÉGALITÉ ET DE GRATUITÉ ?

Auteur(s): $\quad$ Annie CARRIER

Gary MULLINS

Revue : $\quad$ RDUS, 2007-2008, volume 38, numéro 1

Pages: $\quad 87-126$

ISSN : $\quad 0317-9656$

Éditeur : $\quad$ Université de Sherbrooke. Faculté de droit.

URI : $\quad$ http://hdl.handle.net/11143/11560

DOI : https://doi.org/10.17118/11143/11560 
Page vide laissée intentionnellement. 


\title{
ARTICLE
}

\section{L'ACCÈS AUX SERVICES D'AIDE À L'HYGIÈNE EN RÉSIDENCE POUR PERSONNES ÂGÉES : UNE ATTEINTE AUX PRINCIPES D’ÉGALITÉ ET DE GRATUITÉ?}

\author{
par Annie CARRIER* \\ Gary MULLINS**
}

Les principes d'égalité et de gratuité en matière de services de santé, notamment les services de soutien à domicile, ne s'appliquent pas de façon absolue. La réalité juridique et clinique démontre que les choix de l'établissement en matière d'offre de services modulent à la fois l'accessibilité et la gratuité pour certaines clientèles. L'aide à l'hygiène en résidence privée pour personnes âgées en est un bel exemple. En effet, le Centre de santé et de services sociaux n'a pas l'obligation légale de fournir directement ce service ou d'en assurer l'accès gratuit à l'usager habitant une telle résidence. Par ailleurs, les pratiques administratives et cliniques en matière d'accès et de dispensation de l'aide à l'hygiène varient d'un établissement à l'autre. Des questionnements sur l'équité de ces pratiques se posent donc et des pistes de solution sont envisagées.

Free and equal access principles do not apply in an absolute manner to health care services, including those relating to home care. The reality of both legal and clinical considerations demonstrates that access to a service and its financial cost, at least for a certain clientele, is modulated by the health care institution's choices regarding the delivery of health services. A good example of this is bathing assistance in residences for the elderly. Indeed, Health and Social Services centres have no legal obligation to provide this service nor do they have to ensure gratuitous access to clients of privately operated residences for the elderly. In addition, administrative and clinical practices regarding access to and delivery of bathing assistance differ from one institution to another. Certain ethical questions are raised and various likely solutions regarding these practices are examined by the writers.

\footnotetext{
* $\quad$ Ergothérapeute, candidate à la Maîtrise en Droit et Politiques de la Santé de l'Université de Sherbrooke. Elle enseigne à l'École de réadaptation de l'Université de Sherbrooke.

** $\quad$ Avocat-conseil en jurisanté. Il enseigne en droit de la santé à l'Université de Sherbrooke. Les auteurs tiennent à remercier Monsieur Michaël Gagnon, professionnel de recherche à la Faculté de droit de l'Université de Sherbrooke, à la fois pour son aide précieuse dans la recherche bibliographique et pour ses judicieux commentaires.
} 


\section{SOMMAIRE}

Introduction

I - Le contexte législatif et réglementaire de la problématique 93

A - $\quad$ Les grands principes directeurs : les législations canadienne et québécoise ................................. 93

B - $\quad$ La mission C.L.S.C. du C.S.S.S. ......................... 96

C - Les résidences pour personnes âgées ................. 100

II - La problématique : l'aide à l'hygiène ................... 105

A - $\quad$ L'aide à l'hygiène, partie prenante des soins auxquels s'appliquent les principes d'égalité et de gratuité?

$B$ - $\quad$ Les C.S.S.S. ont-ils l'obligation d'offrir les services d'aide à l'hygiène en résidence pour personnes âgées?

III - Questionnement éthique et pistes de solution

Conclusion

Annexe 1

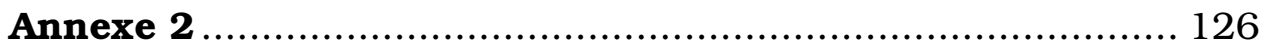




\section{Introduction}

L'organisation des services de santé et des services sociaux a connu de grands bouleversements au cours des cinquante dernières années, nul ne l'ignore. Au départ sous la responsabilité d'intérêts privés, majoritairement de congrégations religieuses, les services de santé, avec la Révolution tranquille et l'émergence de l'État-providence, passent sous la gouverne de l'État ${ }^{1}$.

C'est au cours de la décennie 70 que la Commission Castonguay-Nepveu ${ }^{2}$ émet ses recommandations mettant ainsi en branle une réforme majeure du système de santé et du système professionnel. La Loi sur les services de santé et les services sociaux $^{3}$ crée ainsi les Centres locaux de services communautaires ${ }^{4}$. La crise inflationniste, combinée à une augmentation des demandes en services de santé et à une amélioration des conditions salariales des employés du secteur public, entraîne une hausse fulgurante des dépenses de santé5. L'ensemble du système se devait donc d'être réévalué afin de réduire les dépenses publiques.

1. Voir généralement Pierre Bergeron et France Gagnon, "La prise en charge étatique de la santé au Québec: émergence et transformations" dans Vincent Lemieux et al., dir., Le système de santé au Québec: organisations, acteurs et enjeux, Ste-Foy, Presses de l'Université Laval, 2003, 7 [Pierre Bergeron et France Gagnon, "prise en charge»] (pour un survol historique de la prise en charge étatique du système de santé québécois).

2. Québec, Commission d'enquête sur la santé et le bien-être social, Rapport de la Commission d'enquête sur la santé et le bien-être social, vol. 7, Québec, Éditeur officiel du Québec, 1967 (Présidents : Claude Castonguay et Gérard Nepveu) ; Voir Joseph Facal, Volonté politique et pouvoir médical. La naissance de l'assurance-maladie au Québec et aux ÉtatsUnis, Montréal, Boréal, 2006 (pour une analyse sociologique de la genèse de cette réforme, le lecteur intéressé pourra se référer avec plaisir à l'ouvrage de cet auteur).

3. L.R.Q., c. S-4.2 [L.S.S.S.S.].

4. Ci-après, [C.L.S.C.].

5. Michel Clavet, Ronald Côté et Thomas Duperré, "L'évolution du financement des dépenses québécoises en services de santé" dans Vincent Lemieux et al. dir., Le système de santé au Québec: organisations, acteurs et enjeux, Ste-Foy, Presses de l'Université Laval, 2003 à la p. 378 (Au Canada, les dépenses totales de santé per capita, en dollars US et en parité 
L'accès aux services d'aide à l'hygiène en résidence pour personnes âgées : $\quad$ (2007) 38 R.D.U.S. une atteinte aux principes d'égalité et de gratuité?

Simultanément, la philosophie d'intervention auprès de la clientèle souffrant d'incapacités ou de handicaps variés, tant physiques que mentaux, change. La société fait preuve d'une plus grande ouverture à l'intégration sociale de ces individus et l'État cherche des modalités d'intervention moins "lourdes" 6. On s'oriente alors vers une vague sans précédent de désinstitutionalisation. Le maintien à domicile était né! Les établissements assumant la mission C.L.S.C. se voient confirmer leur rôle de maintien dans le milieu, tant pour les personnes handicapées que pour les personnes âgées. Ils assument un rôle de prévention et de porte d'entrée du réseau.

Vers la fin des années 80 , le problème criant des listes d'attente pour les chirurgies électives, combiné à l'émergence de nouvelles technologies médicales, fait tendre vers l'utilisation de la chirurgie d'un jour ${ }^{7}$. La Commission Rochon ${ }^{8}$ encourage donc l'État à prendre le virage ambulatoire. Cette nouvelle façon de faire entraîne une restructuration complète des services offerts par les C.L.S.C. On assiste à un transfert massif d'infirmières vers ceuxci, dont le rôle premier est d'assurer le suivi à domicile de la clientèle post-opérée. De porte d'entrée, le C.L.S.C. devient porte de sortie!

du pouvoir d'achat, sont passées de $428 \$$ (en 1975), à $710 \$$ (en 1980), à $1193 \$$ (en 1985)).

6. Dans la foulée du mouvement américain "The Independent Living Movement" et de l'Année internationale des personnes handicapées (1981), le Conseil des ministres donne comme mandat à l'Office des personnes handicapées du Québec (O.P.H.Q.) de soumettre une proposition de politique d'ensemble de prévention de la déficience et d'intégration sociale des personnes handicapées. À part... égale ; Québec, L’intégration sociale des personnes handicapées : un défi pour tous, Office des personnes handicapées du Québec, Québec, 1984 (traite notamment des services de maintien à domicile).

7. Canada, Association féminine d'éducation et d'action sociale, Qui donnera les soins? Les incidences du virage ambulatoire et des mesures d'économie sociale sur les femmes du Québec, Condition féminine du Canada, 1998.

8. Québec, Commission d'enquête sur les services de santé et les services sociaux, Rapport de la Commission d'enquête sur les services de santé et les services sociaux, Québec, Publications du Québec, 1988. 
2007) 38 Laccès aux services d'aide à l'hygiène en résidence pour personnes âgées:

une atteinte aux principes d'égalité et de gratuité?

Parallèlement à ces modifications dans les modes d'intervention, l'État vit une crise des finances publiques. Avec le virage viennent les coupures, et les budgets qui devaient suivre les nouvelles responsabilités s'avèrent nettement insuffisants ${ }^{9}$. Mais les C.L.S.C. et les centres hospitaliers ne font pas seuls les frais des coupures et les Centres d'hébergement et de soins de longue durée ${ }^{10}$ passent également au couperet. Leur nombre de lits disponibles diminue, leur clientèle s'alourdit et les critères d'admission deviennent plus sévères ${ }^{11}$. Les résidences privées d'hébergement se multiplient et reçoivent de plus en plus de gens en perte d'autonomie importante.

Un nombre croissant de personnes âgées recourt aux services de ce type de résidence ${ }^{12}$. Cependant, les services offerts et les coûts varient; d'une résidence à l'autre, on note donc des écarts importants ${ }^{13}$. De même, les services des C.L.S.C. offerts aux rési-

9. Michèle Charpentier, Priver ou privatiser la vieillesse? Entre le domicile à tout prix et le placement à aucun prix, Ste-Foy, Presses de l'Université du Québec, 2002, n. 4 [Charpentier, Priver ou privatiser] (les énoncés politiques prévoyaient une hausse du financement des services à domicile de l'ordre de $150 \%$ entre 1990 et 1995 . Les données démontrent plutôt une variation effective de $64 \%$ de ce financement entre 1986 et 1995).

10. Ci-après, [C.H.S.L.D.].

11. Michèle Charpentier, Vieillir en milieu d'hébergement. Le regard des résidents, Québec, Presses de l'Université du Québec, 2007, c. 1 ; Charpentier, Priver ou privatiser, supra note 9 à la p.18 (à titre d'exemple, en 1985 les personnes âgées hébergées en centre d'accueil nécessitaient en moyenne 1,85 heure de soins et d'assistance par jour. Aujourd'hui, la moyenne est de 3,5 heures).

12. Michèle Charpentier, "De l'institution au marché: transformation de l'hébergement des personnes âgées et enjeux actuels" (2004) $3: 2$ Vie et vieillissement 2 à la p. 4 ("ces résidences, en marge du réseau public, constituent désormais l'acteur principal de l'hébergement des personnes âgées au Québec. Le nouveau registre des résidences pour personnes âgées, tenu par les régies (agences régionales), recense actuellement 80000 places (RRSSSMC, 2003), soit deux fois plus que les CHSLD publics et privés réunis").

13. Voir notamment Agence de la santé et des services sociaux de l'Estrie, Registre des résidences avec services pour personnes âgées, Estrie 20062007, Sherbrooke, 2006 [Agence de la santé et des services sociaux de l'Estrie]; Gina Bravo, "Résidences sans permis ministériel : profil des résidants et qualité des soins" dans R. Hébert et R. Kouri, dir., Les échan- 
dents de ces ressources diffèrent d'une région à l'autre, d'une M.R.C. à l'autre et même, d'une partie de la ville à l'autre14.

Ce constat et les problèmes récurrents du système en lien avec l'accessibilité aux services et leur continuité, ont mené le Ministre Couillard et son gouvernement à adopter une nouvelle réforme du réseau de la santé. Le Projet de Loi $83^{15}$ a mené notamment au regroupement d'établissements en un seul, multivocationnel, à savoir le Centre de santé et de services sociaux ${ }^{16}$, cherchant du coup à mettre fin au fonctionnement "en silo". Il a également intégré dans la prestation de soins un grand nombre d'organismes privés à but lucratif, dont les résidences pour personnes âgées. Nous nous attarderons ici sur la question en titre d'un point de vue juridique en traitant tout d'abord du contexte législatif et réglementaire de la problématique. Nous réviserons tout d'abord brièvement les principes directeurs du système de santé, les responsabilités du C.S.S.S., les notions de "résidence pour personnes âgées" et de certification. Nous nous demanderons ensuite si l'aide à l'hygiène fait partie des soins auxquels les principes d'égalité et de gratuité s'appliquent, et si ceux-ci devraient être offerts par le C.S.S.S. en vertu de sa mission C.L.S.C. Nous terminerons en soulevant des questions éthiques en lien avec l'accessibilité aux services d'aide à l'hygiène et en évoquant des pistes de solution possibles. Pour ce faire, nous nous baserons, outre la législation, sur les documents officiels du ministère de la

ges cliniques et scientifiques sur le vieillissement. Actes du Congrès scientifique Autonomie et vieillissement, Institut Universitaire de Gériatrie de Sherbrooke, 1999, 106 [Bravo, "Résidences sans permis»].

14. Effectivement, les C.L.S.C. de Sherbrooke avaient des pratiques différentes. Selon que la personne vivait d'un côté ou de l'autre de la rivière StFrançois, l'aide offerte par le C.L.S.C. desservant le territoire n'était pas la même! La fusion des C.L.S.C. Gaston-Lessard et SOC en 1999 a heureusement permis une uniformisation ultérieure des pratiques. Il convient toutefois de noter qu'en Estrie les pratiques varient toujours d'une M.R.C. à l'autre. Certains établissements offrent les services d'aide à l'hygiène aux personnes le nécessitant, soit par l'entremise de leur personnel ou par achat de services avec la résidence ou avec des entreprises d'économie sociale, d'autres n'offrent pas le service s'il est inclus dans le bail. Nous y reviendrons. 
Santé et des Services sociaux ${ }^{17}$, sur la doctrine et sur la jurisprudence en la matière.

\section{I - Le contexte législatif et réglementaire de la problémati- que}

\section{A - Les grands principes directeurs : les législations cana- dienne et québécoise}

Afin de bien cerner la problématique qui nous occupe, il convient tout d'abord d'identifier clairement son contexte législatif et réglementaire.

Le système de santé relève principalement de la compétence des provinces ${ }^{18}$. C'est cependant suite aux programmes de subventions fédéraux, et particulièrement du programme national d'assurance-hospitalisation de 1957, que la plupart des provinces se sont dotées d'un régime d'assurance-hospitalisation ${ }^{19}$.

Aujourd'hui, la Loi canadienne sur la santé20 régit les éléments conditionnels à l'obtention de l'aide financière du gouvernement fédéral aux provinces, tel que spécifié à l'article 4 :

La présente loi a pour raison d'établir les conditions d'octroi et de versement d'une pleine contribution

15. Loi modifiant la Loi sur les services de santé et les services sociaux et d'autres dispositions législatives, L.Q. 2005, c. 32.

16. Ci-après, [C.S.S.S.].

17. Ci-après, [M.S.S.S.].

18. Loi constitutionnelle de 1867 (R.-U.), 30 \& 31 Vict., c.3, art. 92 par. 7, reproduit dans L.R.C. 1985, app. II, no 5 .

19. Voir notamment Pierre Bergeron et France Gagnon, "prise en charge", supra note 1 (pour en savoir davantage sur l'émergence du système de santé actuel).

20. R.S.C. 1985, c. C-6 (le titre complet de la loi est Loi concernant les contributions pécuniaires du Canada aux services de santé assurés pris en charge par les régimes provinciaux d'assurance-santé et les montants payables par le Canada pour les programmes de services complémentaires de santé, ce qui, à notre avis, reflète plus exactement sa portée restreinte). 
pécuniaire pour les services de santé assurés et les services complémentaires de santé fournis en vertu de la loi d'une province [nos italiques] ${ }^{21}$.

Ces conditions d'octroi sont définies à l'article 7 , soit la gestion publique, l'intégralité, l'universalité, la transférabilité et l'accessibilité. Nous nous attarderons exclusivement à l'universalité et l'accessibilité, desquelles découlent les principes d'égalité et de gratuité qui nous intéressent. L'article 10 de la Loi canadienne sur la santé édicte que :

La condition d'universalité suppose qu'au titre du régime provincial d'assurance-santé, cent pour cent des assurés de la province ait droit aux services de santé assurés par celui-ci, selon des modalités uniformes [nos italiques] ${ }^{22}$.

L'article 12, pour sa part, spécifie ce que l'on entend par accessibilité, notamment :

La condition d'accessibilité suppose que le régime provincial d'assurance-santé: a) offre les services de santé assurés selon des modalités uniformes et ne fasse pas obstacle, directement ou indirectement, et notamment par facturation aux assurés, à un accès satisfaisant par eux à ces services [nos italiques] ${ }^{23}$.

21. Ibid., art. 4 (on entend par 'services complémentaires' de santé "les services définis dans les règlements et offerts aux habitants d'une province, à savoir : a)les soins intermédiaires en maison de repos; b)les soins en établissements pour adultes; c)les soins à domicile; d)les soins ambulatoires»).

22. Ibid., art. 10 (on entend par "assuré», un "habitant d'une province, à l'exception : a)des membres des Forces canadiennes; b) des membres de la Gendarmerie Royale du Canada nommés à un grade; c)des personnes purgeant une peine d'emprisonnement dans un pénitencier, au sens de la Partie I de la Loi sur le système correctionnel et la mise en liberté sous condition; d)des habitants de la province qui s'y trouvent depuis une période de temps inférieure au délai minimal de résidence ou de carence d'au plus trois mois imposé aux habitants par la province pour qu'ils soient admissibles ou aient droit aux services de santé assurés»).

23. Ibid., art. 12 (on entend par "services de santé assurés" les "services hospitaliers, médicaux ou de chirurgie dentaire fournis aux assurés, à 
La Loi canadienne sur la santé nous apporte un certain éclairage sur les principes et sur les éléments auxquels ils s'appliquent. Cependant, il nous faut définir ce qu'est le régime provincial d'assurance-santé afin de répondre à la question qui nous préoccupe.

Deux lois nous intéresseront plus particulièrement, soit la Loi sur l'assurance maladie et la L.S.S.S.S. ${ }^{24}$ La Loi sur l'assurance maladie $^{25}$ spécifie les services assurés par l'État. On entend par services assurés :

les services, médicaments, prothèses, appareils orthopédiques, aides à la locomotion et à la posture, fournitures médicales ou autres équipements, aides visuelles, aides auditives et aides à la communication visés dans l'article 3 [nos italiques] ${ }^{26}$.

La lecture de l'article 3 nous permet de constater que les services assurés n'incluent pas l'aide à l'hygiène à domicile 27 .

On retrouve dans la L.S.S.S.S. les droits des usagers du système de santé et les obligations des établissements envers les bénéficiaires. L'article 5 stipule que :

Toute personne a le droit de recevoir des services de santé et des services sociaux adéquats sur les plans à la fois

l'exception des services de santé auxquels une personne a droit ou est admissible en vertu d'une loi fédérale ou d'une loi provinciale relative aux accidents du travail»).

24. Il est évident que la Loi sur l'assurance hospitalisation et ses règlements définissent une part des services de santé assurés par l'État québécois; cependant, une analyse de celle-ci apporterait peu d'éclairage à la question qui nous occupe, les soins à domicile n'en relevant pas. La même conclusion vaut pour la Loi sur l'assurance médicament.

25. L.R.Q., c. A-29.

26. Ibid., art. 1a).

27. Il ne nous apparaît pas pertinent ici de reproduire l'article 3, s'agissant d'une liste exhaustive de services. 
scientifique, humain et social, avec continuité et de façon personnalisée et sécuritaire [nos italiques] ${ }^{28}$.

Il s'agit donc là de l'énonciation claire d'un droit exigible aux services de santé et aux services sociaux. Cet article doit cependant être interprété à la lumière de l'article 13 de la même loi.

Le droit aux services de santé et aux services sociaux et le droit de choisir le professionnel et l'établissement prévus aux articles 5 et 6 , s'exercent en tenant compte des dispositions législatives et réglementaires relatives à l'organisation et au fonctionnement de l'établissement ainsi que des ressources humaines, matérielles et financières dont il dispose [nos italiques] 29.

Le droit aux services n'est donc pas un droit absolu de recevoir tous les soins requis, quel qu'en soit le coût et quels que soient les services disponibles.

\section{B - La mission C.L.S.C. du C.S.S.S.}

La L.S.S.S.S. régit tous les établissements de santé du réseau québécois, qu'ils soient publics ou privés. La réforme mise en place par le ministre Couillard en 2003 par la création des agences de développement de réseaux locaux de services de santé

28. L.S.S.S.S., supra note 3, art. 5 (L'article 6 pour sa part confère au bénéficiaire le droit de choisir le professionnel et l'établissement desquels il désire recevoir des services).

29. Ibid., art. 13 ; Patrick A. Molinari, "L'accès aux soins de santé : réflexion et fondements juridiques de l'exclusion" dans Les droits de la personne et les enjeux de la médecine moderne, Ste-Foy, Presses de l'Université Laval, 1996 à la p. 47 [Patrick A. Molinari, "L'accès"] (D'aucuns voient dans l'article 13 une restriction importante du droit aux services de l'usager. "À la façon d'une peau de chagrin, le droit aux services de santé rétrécirait dès lors que le débiteur de l'obligation de fournir les soins estimerait ne pas avoir les moyens de les offrir". Les jugements plus récents portant sur l'article 13 n'ont pas remis en cause les interprétations antérieures ayant servi de base à l'analyse de Molinari). 
et de services sociaux ${ }^{30}$, devenues, depuis, des agences de santé et de services sociaux, a donné le ton à une nouvelle organisation dans la prestation des services. Le projet d'intégration des services visait "(l)'accès à des services continus et de qualité, quel que soit le territoire de résidence» ${ }^{31}$ de la personne. Pour se faire, la mise en place d'un réseau local de services de santé et de services sociaux ${ }^{32}$ a été privilégiée. La mise en place de ce R.L.S. "vise à responsabiliser tous les intervenants de ce réseau afin qu'ils assurent de façon continue, à la population du territoire de ce réseau, l'accès à une large gamme de services de santé et de services sociaux généraux, spécialisés et surspécialisés" [nos italiques] ${ }^{33}$.

$\mathrm{Au}$ cœur de ces réseaux locaux, l'instance locale, «laquelle est un établissement multivocationnel qui exploite notamment un centre local de services communautaires, un centre d'hébergement et de soins de longue durée et, le cas échéant, un centre hospitalier de soins généraux et spécialisés»"34. C'est cette instance locale qui est responsable de définir le projet clinique et organisationnel $^{35}$ pour le territoire local ${ }^{36}$. Elle est également dûment manda-

30. Loi sur les agences de développement de réseaux locaux de services de santé et de services sociaux, L.R.Q., c. A-8.1.

31. Québec, Ministère de la Santé et des Services Sociaux, L'intégration des services de santé et des services sociaux : le projet organisationnel et clinique et les balises associées à la mise en ouvre des réseaux locaux de services de santé et de services sociaux, Québec, 2004 à la p.1, en ligne: M.S.S.S.

<http://publications.msss.gouv.qc.ca/acrobat/f/documention/2004/04009-08.pdf > [M.S.S.S., L’intégration des services de santé].

32. Ci-après [R.L.S.] ; L.S.S.S.S., supra note 3, art. 99.2 (réfère à "tout réseau mis en place conformément à un décret du gouvernement pris en application de la Loi sur les agences de développement des réseaux locaux de services de santé et de services sociaux (...)").

33. Ibid., art. 99.3 (on parle donc ici d'une responsabilité "populationnelle» et non plus d'une responsabilité face à la seule clientèle demandant des services).

34. Ibid., art. 99.4 (l'instance locale peut seule faire usage, dans son nom, des mots "centre de santé et de services sociaux").

35. Ibid., art. 99.4 (on entend par projet clinique et organisationnel l'identification des besoins sociosanitaires et des particularités de la population du territoire local, des objectifs poursuivis dans l'amélioration de la santé et du bien-être de cette population, de l'offre de services pour 
tée pour exercer la coordination des services ${ }^{37}$ et en être imputable ${ }^{38}$. Le C.S.S.S. doit ainsi assurer l'accès aux services dévolus par ses missions propres mais également, à l'ensemble des services nécessaires à sa population, qu'ils soient de première ligne, de deuxième ou de troisième ligne, par le biais d'ententes de servi$\operatorname{ces}^{39}$ avec les autres prestataires de services ${ }^{40}$.

L'article 80 de la L.S.S.S.S. nous dit que :

La mission d'un centre local de services communautaires est d'offrir en première ligne des services de santé et des services sociaux courants et, à la population du territoire qu'il dessert, des services de santé et des services sociaux de nature préventive ou curative, de réadaptation ou de réinsertion.

À cette fin, l'établissement qui exploite un tel centre s'assure que les personnes qui requièrent de tels services pour elles-mêmes ou pour leurs familles soient rejointes, que leurs besoins soient évalués et que les services requis leur soient offerts à l'intérieur de ses installations ou dans

satisfaire les besoins et les particularités susmentionnés et des modes d'organisation et des contributions attendues des différents partenaires) ; Pour la notion de partenaires, voir infra note 40 (ici, cette notion s'étend aux intervenants des autres secteurs d'activités ayant un impact sur les services de santé et les services sociaux).

36. La notion de territoire local n'est pas définie. Les directives ministérielles réfèrent toutefois au territoire desservi par le C.L.S.C. mais d'autres découpages sont aussi possibles, selon les régions.

37. L.S.S.S.S., supra note 3, art. 79 et ss., 99.4-99.7 (cette coordination de services réfère à l'idée de leur hiérarchisation, à savoir la complémentarité des services de première, deuxième et troisième lignes. La définition de ces notions ne se retrouve pas comme tel dans la L.S.S.S.S., où on parle plutôt de la mission des établissements offrant des services généraux, spécialisés et surspécialisés).

38. Ibid., art. 182.1-182.8.

39. Ibid., art. 99.7(2).

40. Ibid. (les autres prestataires ou partenaires sont "notamment, les établissements offrant des services spécialisés et surspécialisés, les médecins du territoire, les organismes communautaires, les entreprises d'économie sociale et les ressources privées") ; M.S.S.S., L'intégration des services de santé, supra note 31 aux pp.7-11 (selon les directives ministérielles, les résidences pour personnes âgées en perte d'autonomie sont incluses dans les ressources privées). 
(2007) 38 R.D.U.S. L'accès aux services d'aide à l'hygiène e résidence pour personnes âgées:

une atteinte aux principes d'égalité et de gratuité?

leur milieu de vie, à l'école, au travail ou à domicile ou, si nécessaire, s'assure qu'elles soient dirigées vers les centres, les organismes ou les personnes les plus aptes à leur venir en aide.

La mission d'un tel centre est également de réaliser des activités de santé publique sur son territoire, conformément aux dispositions prévues dans la Loi sur la santé publique (chapitre S-2.2)" [nos italiques] ${ }^{41}$.

Comme tout établissement, l'établissement responsable de la mission d'un C.L.S.C. doit déterminer les services de santé et les services sociaux qu'il dispense, en tenant compte de cette mission et des ressources disponibles ${ }^{42}$. Il peut édicter des règlements 43 et doit notamment le faire dans les cas prévus à l'article 6 paragraphes 2 et $14 \mathrm{du}$ Règlement sur l'organisation et l'administration des établissements ${ }^{44}$.

41. L.S.S.S.S., supra note 3, art 80 (il est à noter que la L.S.S.S.S. ne comprend pas de section consacrée aux définitions. L'étendue des termes "services de santé" et "services sociaux" peut cependant être induite de l'article 1 de ladite loi qui cerne les objectifs de celle-ci, notamment: maintenir et améliorer la capacité physique, psychique et sociale des personnes d'agir dans leur milieu et d'accomplir leurs rôles; favoriser l'adaptation ou la réadaptation des personnes, leur intégration ou leur réintégration sociale; diminuer l'impact des problèmes qui compromettent l'équilibre, l'épanouissement et l'autonomie des personnes. On peut également se demander ce que l'on entend par "domicile». S'agit-il ici de l'endroit où demeure l'usager, sans distinction? D'une résidence temporaire ou permanente? Peut-on y inclure la résidence pour personnes âgées? Doit-on ici appliquer la notion de domicile du Code civil du Québec? Rien n'est moins sûr puisque l'article 75 ne s'applique que dans l'exercice des droits civils. Or, le droit aux services de santé est un droit social qui n'a pas la même portée qu'un droit civil) ; Voir Andrée Lajoie, "L'émergence d'un droit social : le droit aux services" dans Pour une approche critique du droit de la santé, Mtl., Les Presses de l'Université de Montréal, 1987 à la p. 21 [Andrée Lajoie, "L'émergence"](La Politique de soutien à domicile du M.S.S.S. spécifie plus clairement ce que sont ces services, de même que la notion de domicile; nous y reviendrons).

42. L.S.S.S.S., supra note 3, art. 105.

43. Ibid., art. 106.

44. Règlement sur l'organisation et l'administration des établissements, c. S-5, r.3.01 art. 6(2), (14) (le conseil d'administration de l'établissement doit réglementer les points suivants soit, selon le paragraphe 2 , les orientations et les activités de l'établissement; selon le paragraphe 14, pour la 


\section{C - Les résidences pour personnes âgées}

Il convient de définir ce qu'est une résidence pour personnes âgées ${ }^{45}$. La L.S.S.S.S. définit cette notion ainsi :

Une résidence pour personnes âgées est un immeuble d'habitation collective où sont offerts, contre le paiement d'un loyer, des chambres ou des logements destinés à des personnes âgées et une gamme plus ou moins étendue de services, principalement reliés à la sécurité et à l'aide à la vie domestique ou à la vie sociale, à l'exception d'une installation maintenue par un établissement et d'un immeuble ou d'un local d'habitation où sont offerts les services d'une ressource intermédiaire ou d'une ressource de type familial ${ }^{46}$.

mission C.L.S.C., la détermination des critères d'accès aux services par des groupes de personnes).

45. Ces résidences excluent un établissement au sens de la L.S.S.S.S., tel que nous l'exposerons un peu plus loin.

46. L.S.S.S.S., supra note 3, art. 5 (ceci constitue la reconnaissance de l'existence de telles ressources. Il s'agit cependant d'une définition large pouvant inclure un nombre important d'installations) ; Québec, Ministère de la Santé et des Services Sociaux, Chez-soi : le premier choix. Précisions pour faciliter l'implantation de la Politique de soutien à domicile, Québec, 2004 à la p. 6 [Ministère de la santé et des services sociaux, Chez soi] (cependant, le Ministère spécifie que "(l)es catégories de résidences pour personnes âgées visées par cet article de loi sont les suivantes : les résidences privées à but lucratif, les résidences privées à but non lucratif, les coopératives d'habitation avec services, les habitations à loyer modique avec services et les communautés religieuses». Il faut noter par ailleurs que la loi ne définit nullement ce qu'on entend par "personnes âgées") ; CSSS-IUGS, Le projet clinique du RLS de Sherbrooke, volet PALV 20062009 : Fiche-synthèse (section 5), 2006 à la p. 2 (s'agit-il d'un état fondé exclusivement sur l'âge ou peut-on penser que le niveau d'autonomie de la personne doit être considéré? Par exemple, l'offre de services du RLS volet PALV de Sherbrooke définit sa clientèle cible, en accord avec les orientations régionales, comme étant les "(p)ersonnes qui sont en perte d'autonomie principalement due à l'avancement en âge, et ce, peu importe la cause : perte d'autonomie fonctionnelle, problèmes cognitifs ou maladies chroniques. Inclut les personnes de moins de 65 ans qui ont les mêmes besoins que celles de 65 ans et plus aux prises avec le même problème"). 
2007) 38 Laccès aux services d'aide à l'hygiène en résidence pour personnes âgées:

une atteinte aux principes d'égalité et de gratuité?

Les services offerts par ce type de résidence ne seraient donc pas exclusivement de nature domestique ou sociale et pourraient inclure, des services d'aide à la vie quotidienne, notamment l'aide à l'hygiène ${ }^{47}$. L'Agence de la santé et des services sociaux ${ }^{48}$ de la région, en vertu des articles 346.0.1 et suivants, est tenu de constituer et de maintenir à jour un registre de ces installations et d'émettre les certificats de conformité demandés par l'exploitant de ces ressources.

En effet, tout exploitant d'une résidence privée pour personnes âgées doit être titulaire d'un tel certificat qui atteste que ladite résidence remplit les conditions exigées ${ }^{49}$. Ces exigences varieront selon que la résidence privée offre ou non des services d'assistance personnelle. Le défaut de se conformer à cette obligation constitue une infraction pénale susceptible d'encourir des amendes ${ }^{50}$.

47. Agence de développement de réseaux locaux de services de santé et de services sociaux Estrie, Programme-services Perte d'autonomie liée au vieillissement (PALV) : contexte, état de situation régional, orientations régionales, plan régional de mise en ouvre, Estrie 2005-2010, 2005 à la p. 56 [Agence de développement de réseaux locaux de services de santé et de services sociaux Estrie, Programme-services] (en ce sens, l'Agence de la santé et des services sociaux de l'Estrie spécifie que "(l)a nouvelle version du registre des résidences pour personnes âgées 2005-2006 (...) inclura l'identification de cinq types de services disponibles pour la clientèle : services de repas, soins d'assistance personne, aide à l'alimentation, soins infirmiers et services de loisirs").

48. Ci-après, [l'Agence].

49. L.S.S.S.S., supra note 3, art. 346.0.3-346.0.4 (ces conditions sont de se conformer aux critères sociosanitaires déterminés par règlement et de satisfaire aux exigences identifiées par règlement. Il convient de souligner que ce certificat ne constitue pas une délivrance de permis d'exploitation au sens de l'article 437 et que donc, les résidences pour personnes âgées ne peuvent être assimilées à un centre au sens de la loi. Ce faisant, elles ne peuvent être considérées comme débitrices des obligations découlant des articles 5 et 6 . Elles demeurent régies en grande partie, comme nous le verrons plus loin, par le Code civil du Québec).

50. Ibid., art. 531.1. L'entrée en vigueur de cette disposition est prévue le 1er février 2009 (bien que les agences réclament depuis longtemps de meilleurs outils de contrôle, on ignore encore comment celles-ci rendront ces nouveaux outils véritablement efficients); Ibid., art. 346.0 .3 al. 2 (il convient néanmoins de souligner un incitatif pour les exploitants de tel- 


\section{L'article 83 définit pour sa part la mission d'un C.H.S.L.D.,} soit :

d'offrir de façon temporaire ou permanente un milieu de vie substitut, des services d'hébergement, d'assistance, de soutien et de surveillance ainsi que des services de réadaptation, psychosociaux, infirmiers, pharmaceutiques et médicaux aux adultes qui, en raison de leur perte d'autonomie fonctionnelle ou psychosociale, ne peuvent plus demeurer dans leur milieu de vie naturel, malgré le support de leur entourage [nos italiques] ${ }^{51}$.

Or, l'article 437 fait part d'une interdiction formelle d'exercer ces activités pour quiconque ne détient pas un permis délivré par le ministre. Cette interdiction est assortie de mesures de contrôle exercées par le ministre et l'Agence concernée ${ }^{52}$. Une résidence pour personnes âgées n'exercerait donc pas les activités propres à un C.H.S.L.D. ${ }^{53}$.

La notion de résidence privée a fait l'objet de recherches dans le domaine de la santé54. Selon Charpentier, "les résidences

les résidences à se conformer à la certification. En effet, "(u)n établissement public, doit, avant de proposer à un usager une résidence pour personnes âgées, s'assurer que l'exploitant de cette résidence est titulaire d'un tel certificat»).

51. Ibid., art. 83.

52. Voir généralement Ibid., art. 452 (concernant la relocalisation des personnes hébergées) ; Ibid., art.489 (concernant l'inspection) ; Charpentier, Priver ou privatiser, supra note 9 aux pp. 69-71 (il s'agit somme toute de pouvoirs limités et peu utilisés).

53. L.S.S.S.S., supra note 3, art. 454 (il apparaît cependant à l'article 454 de la L.S.S.S.S. qu'une résidence privée d'hébergement, notion qui n'est pas comme telle définie, pourrait obtenir des allocations financières pour offrir des services à des personnes en perte d'autonomie. Nous y reviendrons).

54. Voir généralement François Aubry, "Les résidences privées à but lucratif pour personnes âgées: un portrait sectoriel» dans Yves Vaillancourt et Michèle Charpentier, dir. Cahiers du LAREPPS, no 05-08, UQAM, 2005 ; Yves Vaillancourt et Michèle Charpentier dir., Les passerelles entre l'État, le marché et l'économie sociale dans les services de logement social et d'hébergement pour les personnes âgées, LAREPPS, UQAM, 2005 (pour une analyse de la contribution des secteurs public (l'État), privé (le mar- 
2007) Laccès aux services d'aide à l'hygiène en

résidence pour personnes âgées :

une atteinte aux principes d'égalité et de gratuité?

pour personnes âgées sont des entreprises privées, généralement à but lucratif, qui appartiennent au secteur marchand de l'hébergement»55. Or, "les rapports entre les propriétaires et les résidants sont régis par les dispositions générales du Code civil en matière de contrat, de louage de choses, plus spécifiquement de bail d'un logement»56. Les règles usuelles en matière de bail de location s'appliquent aux résidences privées. La Régie du logement peut donc intervenir en cas de litige ${ }^{57}$. Si, en raison de l'âge ou du handicap du locataire, des services additionnels sont offerts, une

ché) et social (les entreprises d'économie sociale) en matière de logement social et d'hébergement des personnes âgées).

55. Michèle Charpentier, "Le droit et les rapports de dépendance vécus par les aînés : le cas des résidences privées pour personnes âgées", Rapport final Commission du droit du Canada, 1999 à la p. 22 ; Charpentier, Priver ou privatiser, supra note 9 à la p. 31 (une définition ultérieure de la même auteure détaille davantage la notion de résidence privée en émettant qu'il s'agit d'"une ressource d'habitation collective de type chambre et pension (...), accueillant au moins deux personnes âgées, appartenant à une personne morale ou physique et qui offre une gamme plus ou moins étendue de services (...)») ; Ibid (la structure d'habitation pour â̂nés ne suffisant pas pour être considérée résidence pour personnes âgées, la ressource, pour être qualifiée de telle, doit donc offrir des services d'hébergement de base tels gîte, couvert, surveillance et ne pas être rattachée au réseau public, c'est-à-dire agréée. L'agrément des ressources privées est possible en vertu des articles 454 à 462 de la L.S.S.S.S.)

56. Charpentier, Priver ou privatiser, Ibid. à la p. 23 (On fait référence ici plus particulièrement aux articles 1892 à 1978).

57. L.S.S.S.S., supra note 3, art. 60 (4.1) (il est important ici de souligner que les nouvelles dispositions de la L.S.S.S.S. permettent dorénavant au résidant d'une résidence privée pour personnes âgées de déposer une plainte auprès de l'agence de son territoire de résidence relativement à des "services qu'elle a reçus ou aurait dû recevoir". Cette plainte sera transmise au Commissaire régional à la qualité des services) ; Jean-Pierre Ménard, "Après le projet de loi 83 : enjeux cachés et droits nouveaux pour les usagers du système de santé" dans Après le projet de Loi 83: Un nouveau réseau de la santé, Collection Développements récents 260, Service de formation continue du Barreau du Québec, 2006 à la p. 26 (Cependant, "dès lors qu'une plainte du résident découle de l'application du bail, le commissaire local est sans juridiction, puisque la Régie du logement a compétence exclusive. (...) Le commissaire local trouve sa juridiction dans les obligations de l'exploitant que conditionnent (sic) sa certification"). 
annexe doit être ajoutée au bail58. Cette annexe comprend une description détaillée de l'aménagement du logement et une liste de services pouvant être dispensés, inclus dans le prix du loyer ou avec supplément (montant mensuel fixe ou prix à la carte).

Dans la région de l'Estrie 59 , les services offerts, de même que les coûts sont variables. Cependant, il appert que $80 \%$ des résidences certifiées ont déclaré offrir les soins d'assistance personnelle (notamment, l'aide à l'hygiène)60. Une revue nonexhaustive des contrats-types proposés par ces résidences ${ }^{61}$ nous

58. Voir Règlement sur les formulaires de bail obligatoires et sur les mentions de l'avis au nouveau locataire, D.907-96, 1996, 128 G.O.Q. II. 4855 (les obligations de l'exploitant en termes de services d'assistance personnelle incorporés au bail pourraient ainsi relever de la compétence de la Régie du logement et non pas du Commissaire régional).

59. Charpentier, Priver ou privatiser, supra note 9 à la p.89 (nous nous attardons spécifiquement à la région de l'Estrie pour deux raisons. Tout d'abord, "(l)'Estrie, notamment par sa réalité biculturelle, est considérée comme une région représentative du Québec. À preuve, on y effectue souvent des études de marché avant d'étendre un produit à l'échelle de la province ou du pays (...). Plusieurs indices tendent à démontrer que la situation des résidences privées en Estrie ne diffère pas de celle des autres régions : la répartition du nombre de lits d'hébergement dans le réseau sociosanitaire y est similaire, le cadre législatif et les politiques ministérielles régissant les résidences privées s'appliquent à l'ensemble du Québec". La pratique clinique en tant qu'ergothérapeute de l'auteure s'y est déroulée sur une période de plus de dix ans, ce qui en fait un milieu à la réalité bien connue).

60. Agence de la santé et des services sociaux de l'Estrie, supra note 13 ; Bravo, "Résidences sans permis", supra note 13 (l'étude de Bravo et coll. nous démontrait d'ailleurs que $73 \%$ des résidences offraient des services d'aide à l'hygiène et que $17 \%$ des résidants pouvaient être qualifiés de "personnes en lourde perte d'autonomie»). CSSS-IUGS, Le projet clinique du RLS de Sherbrooke volet PALV 2006-2009 : faits saillants (section 6), 2006 à la p. 5 (pour la M.R.C. de Sherbrooke, "près de 3700 personnes vivent dans une résidence privée dont près de 600 dans une communauté religieuse*; $38 \%$ ont 85 ans et plus*; près de $50 \%$ des résidents de ces milieux ont des incapacités modérées à sévères»).

61. Cette revue est basée sur la pratique clinique de l'auteure en tant qu'intervenant responsable des plans d'intervention et des plans de services individualisés et membre de l'équipe d'accueil-évaluationinformation-orientation-référence (AEIOR) de la mission C.L.S.C. du 
L'accès aux services d'aide à l'hygiène en
(2007) 38 R.D.U.S. résidence pour personnes âgées :
une atteinte aux principes d'égalité et de gratuité?

a vite permis de conclure que la plupart de ces services sont habituellement fournis moyennant une contribution supplémentaire. Cette courte description du contexte légal et réglementaire de la question qui nous occupe nous permet maintenant de répondre à notre première interrogation : l'aide à l'hygiène fait-elle partie des services auxquels s'appliquent les principes d'égalité et de gratuité?

\section{II - La problématique : l'aide à l'hygiène}

\section{A - L'aide à l'hygiène, partie prenante des soins auxquels s'appliquent les principes d'égalité et de gratuité?}

Pour répondre à cette question, il convient tout d'abord de se rapporter aux textes législatifs de la première partie. La Loi canadienne sur la santé est claire : les services de santé assurés et donc gratuits sont les services hospitaliers, médicaux ou de chirurgie dentaire. Les soins à domicile, y compris l'aide à l'hygiène, font partie des services complémentaires de santé. Or, ceux-ci n'ont pas à être assurés pour répondre aux conditions d'octroi des paiements de transferts. Ceci n'exclut cependant pas qu'ils puissent tout de même être "assurés" par le régime d'assurance santé de la province visée.

Or, au Québec, les lois actuelles, soit la Loi sur l'assurance maladie, la Loi sur l'assurance hospitalisation et la Loi sur l'assurance médicament, définissent les services "assurés". La L.S.S.S.S., pour sa part, énonce un droit aux services de santé et aux services sociaux $\underline{\text { exigible }}^{62}$. Il ne faut cependant pas confondre

CSSS-IUGS, de même que sur son implication dans le Comité Mécanisme d'accès à l'hébergement de la M.R.C. de Sherbrooke.

62. Voir Andrée Lajoie, "L'émergence", supra note 41 ; Dominique Sprumont, "Le droit d'accès aux services de santé en droit québécois" (1998) 6 Health L. J. à la p. 203 [Dominique Sprumont, "Le droit d'accès»]; Patrick A. Molinari, "L'accès", supra note 29 (il est intéressant de noter que la Loi canadienne sur la santé ne fait nullement d'une telle exigibilité une condition d'octroi de subvention; le Québec est allé beaucoup plus loin que ce qui était nécessaire à l'obtention des paiements de transferts. Ce "débordement" semble en lien, pour certains auteurs, avec la survalorisa- 
ce droit avec le droit à la gratuité. Les services exigibles ne sont donc pas nécessairement gratuits. Le jugement $R$. Dagenais $c$. C.L.S.C. Kateri vient d'ailleurs corroborer cette analyse ${ }^{63}$.

La Loi sur les services de santé et les services sociaux confère aux bénéficiaires certains droits et avantages comme des services de santé exigibles des C.L.S.C. s'ils sont mentionnés au plan d'organisation du C.L.S.C. et dans la mesure des ressources disponibles. Aucune disposition ne permet cependant de prétendre à la gratuité des services. D'autre part, la loi ne prévoit pas qu'un C.L.S.C. ne puisse exiger des bénéficiaires des frais pour certains services qu'il offre. Les règlements adoptés en vertu de la loi ne contiennent pas non plus de normes concernant la gratuité ou non des services offerts par un C.L.S.C. ${ }^{64}$

En effet,

(...) la gratuité des services de santé garantie par les programmes fédéraux et québécois d'assurance santé n'implique pas nécessairement un droit (exigible) d'accès à ces services, l'inverse n'étant pas non plus vrai. Le droit d'accès aux services de santé n'a effectivement pas été admis comme fondement éventuel d'un droit à la gratuité de ces services. 65

Il apparaît donc que rien dans la législation actuelle n'assure la gratuité des services de soins à domicile66. Par contre, il nous semble que la notion d'égalité soit protégée par la loi qué-

tion de l'État propre au Québec des années soixante et soixante-dix. Il convient ici de rappeler que le droit aux services est soumis aux limites édictées par l'article 13 de la L.S.S.S.S. et n'est donc pas absolu).

63. R. Dagenais c. C.L.S.C. Kateri [1992] R.L. 395 (C.A. Qué.).

64. Ibid. à la p. 397.

65. Dominique Sprumont, "Le droit d'accès", supra note 62 à la p. 203.

66. L.S.S.S.S., supra note 3, art. 99.3 (la notion de responsabilité populationnelle se limite d'ailleurs à assurer «l'accès à une large gamme de services de santé et de services sociaux généraux" [nos italiques]). 
bécoise. En effet, l'article 5 de la L.S.S.S.S. édicte clairement que toute personne a droit de recevoir des services de santé67.

Que la gratuité ne soit pas "assurée" pour les services de soins à domicile ne veut pas dire pour autant que les gens déboursent pour les recevoir. Au contraire, la politique du M.S.S.S. 68 établit que les services à domicile sont fournis gratuitement aux personnes les nécessitant 69 . Cependant, d'un côté, cette politique n'a pas force de loi ou de règlement, comme nous le rappelle le jugement cité précédemment et de l'autre, les précisions apportées dans un document ultérieur limitent l'étendue de cette gratuité ${ }^{70}$.

Il semblerait donc que le principe de gratuité ne serait pas protégé en ce qui a trait aux services à domicile. Un questionnement demeure face à la protection du principe d'égalité d'accès, compte tenu de la limite imposée par l'article 13. Il convient cependant de poursuivre notre réflexion en nous demandant si les C.S.S.S., en vertu de leur responsabilité populationnelle, doivent

67. Cette notion d'égalité a une portée limitée puisqu'elle doit être interprétée avec la réserve de l'article 13. Nous reviendrons un peu plus loin sur l'applicabilité des Chartes en regard de l'égalité d'accès aux soins de santé.

68. Québec, Ministère de la Santé et des Services sociaux, Pour faire les bons choix. Chez-soi : le premier choix. Politique de soutien à domicile, Québec, 2002 [Ministère de la Santé et des Services sociaux, Pour faire les bons choix].

69. Ibid. à la p. 17 (on distingue les services professionnels, qui sont offerts gratuitement pour tous, des services d'aide domestique et d'assistance personnelle qui sont offerts sans frais aux personnes souffrant d'incapacité temporaire, d'incapacité significative et persistante ou en phase palliative) ; Ministère de la santé et des services sociaux, "Chez soi», supra note 46 aux pp. 11-12 (pour une définition des notions d'incapacité temporaire et d'incapacité significative et persistante).

70. Ministère de la santé et des services sociaux, "Chez soi", Ibid. à la p. 11 ("Le principe de gratuité, sur lequel s'appuie la Politique de soutien à domicile, notamment en ce qui concerne les services d'aide à la vie domestique et d'assistance personnelle pour les personnes qui en ont besoin en raison de leur état de santé ou de leur condition, se heurte d'une part aux ressources limitées et d'autre part à la diversité des modalités de prestation de services ou des programmes" [notre soulignement]). 
ou non offrir ces services en résidence pour personnes âgées, et si tel était le cas, sous quelles modalités.

\section{B - Les C.S.S.S. ont-ils l'obligation d'offrir les services d'aide à l'hygiène en résidence pour personnes âgées?}

Nous avons déjà attiré l'attention sur la disparité des services offerts par les C.S.S.S. au sein de la région de l'Estrie. Certains C.S.S.S. offrent le service d'aide à l'hygiène à la personne vivant en résidence privée au même titre que toute personne vivant à domicile, par le biais de son personnel ou par achat de services. Cependant, certains le font s'il s'agit d'une clientèle en très grande perte d'autonomie ${ }^{71}$ seulement. D'autres l'offrent par achat de services uniquement, soit avec une entreprise d'économie sociale, soit avec la résidence pour personnes âgées. Pour d'autres, dès que le service est fourni par la résidence, la personne ne peut avoir accès aux services de l'établissement.

La politique du M.S.S.S. spécifie clairement que le domicile est "(l)e lieu où loge une personne, de façon temporaire ou permanente. Toute personne qui habite dans une maison individuelle, un logement, une résidence collective ou une résidence dite "privée" est admissible au soutien à domicile" [notre soulignement] ${ }^{72}$. Cependant, "(l)'évaluation des besoins et le plan d'intervention ou le plan de services individualisé tiendront compte des services

71. Voir notamment R. Hébert et al., "Le système de mesure de l'autonomie fonctionnelle (SMAF)" dans R. Hébert et K. Kouri, dir., Les échanges cliniques et scientifiques sur le vieillissement. Actes du Congrès scientifique Autonomie et vieillissement, Institut Universitaire de Gériatrie de Sherbrooke, 1999, 86 (le niveau de perte d'autonomie est habituellement déterminé par une évaluation clinique fondée sur l'utilisation du SMAF).

72. Ministère de la Santé et des Services sociaux, Pour faire les bons choix, supra note 68 à la p. 16 ; Ibid. à la p. 5 (de plus, "(l)a définition du domicile doit contribuer à harmoniser les pratiques pour faire en sorte que toutes les personnes soient traitées de façon équitable, peu importe leur domicile ou leur lieu de résidence») ; Ibid. à la p. 6 (et aussi que, "le ministère considère que ce type de résidences (résidence privée) constitue le domicile des personnes qui y résident et qu'à ce titre, celles-ci ont droit à des services de santé et à des services sociaux adéquats offerts de façon continue et personnalisée»). 
fournis par ces résidences" ${ }^{73}$. Il semble donc que la directive administrative du Ministère cautionne les établissements qui n'offrent pas de services dans les résidences où l'aide à l'hygiène fait partie du panier de services ou encore, qui ne l'offrent que par le biais d'achat de services ${ }^{74}$. Il n'est pas clair si le plan de services individualisés doit tenir compte ou non d'éventuels frais supplémentaires pour l'usager qui a recours aux services de la résidence $^{75}$. Or, comme nous l'avons déjà noté, la plupart des résidences offrent des services d'aide à l'hygiène moyennant des frais supplémentaires.

L'aide à l'hygiène offerte dans le cadre de la mission C.L.S.C. est habituellement dispensée par des auxiliaires familiales et sociales ${ }^{76}$ ou des travailleurs gré-à-gré ${ }^{77}$. Ce service, bien

73. Ibid. à la p. 16 ; Québec, Ministère de la Santé et des Services Sociaux, Cadre de référence sur les services à domicile de première ligne, Québec, 1995 (notons qu'auparavant, les résidences privées étaient "considérées comme des domiciles seulement pour les services non couverts dans les baux ou contrats convenus entre les promoteurs ou les propriétaires et les locataires". Il nous apparaît que, dans les faits, la situation soit demeurée la même!).

74. D'ailleurs, la responsabilité de l'instance locale demeure celle d'assurer l'organisation et la coordination des services qui répondent aux besoins de sa population, responsabilité dont elle s'acquitte, notamment, en concluant des ententes de services avec ses partenaires, rappelons-le.

75. Ministère de la santé et des services sociaux, Chez soi, supra note 46 à la p. 6 (sur ce point d'ailleurs, les précisions du Ministère ne sont pas d'une limpidité absolue. On nous dit que "l'instance locale est responsable d'évaluer les besoins des personnes vivant en résidences privées et de s'assurer qu'elles reçoivent les services adaptés à leur état de santé ou à leur condition. Lorsque des services additionnels, à ceux offerts par la résidence privée contre paiement, sont requis, l'instance locale doit offrir les services selon les modalités convenues avec les personnes comme pour l'ensemble de la clientèle ayant besoin de services de soutien à domicile" [nos italiques]. S'agit-il ici pour l'instance locale d'offrir les services qui ne peuvent l'être par la résidence ou d'offrir les services pour lesquels la personne serait tenue de débourser si elle faisait affaire avec la résidence?).

76. Il s'agit d'employés détenant une formation de niveau post-secondaire d'une durée de 6 à 12 mois. Aucun ordre professionnel ne les régit. À noter que dans certains C.L.S.C., des infirmières auxiliaires dispensent les soins d'hygiène; il s'agit cependant d'une pratique peu courante. 
L'accès aux services d'aide à l'hygiène en résidence pour personnes âgées : $\quad$ (2007) 38 R.D.U.S. une atteinte aux principes d'égalité et de gratuité?

que "non-assuré", est pourtant offert gratuitement à la population qui en a besoin et qui habite toujours dans son domicile privé. Or, restreindre l'accès à ce service à une partie de la population sous prétexte que celui-ci fait partie d'un contrat pour lequel elle doit débourser, n'est-ce pas là une atteinte au principe d'égalité découlant de l'article 578 ? On rétorquera alors que la limite de l'article 13 permettrait, sous réserve du plan d'organisation et de fonctionnement de l'établissement tel qu'adopté par son conseil d'administration en vertu des articles 105 et 106 de la L.S.S.S.S. et de l'article 6 du Règlement sur l'organisation et l'administration

77. Ministère de la santé et des services sociaux, Chez soi, supra note 46 à la p. 28 (payé par le C.L.S.C. par le biais du Programme d'allocation directe/Chèque Emploi-Service (CES). Ce programme s'adresse à la clientèle suivante : personnes âgées en perte d'autonomie et personnes handicapées, dont notamment, l'état est stable, les besoins sont à long terme, la situation ne nécessite pas une expertise particulière et qui choisissent de façon libre et éclairée cette option. La pratique de certains établissements de n'offrir l'aide à l'hygiène à la clientèle vivant en résidence pour personnes âgées que selon la seule modalité d'achat de services contreviendrait donc à cette directive).

78. Agence de développement de réseaux locaux de services de santé et de services sociaux Estrie, Programme-services, supra note 47 à la p. 16 (sans compter que l'article 83 décrivant la mission de C.H.S.L.D. fait une distinction entre milieu de vie substitut, soit le centre offrant une mission de C.H.S.L.D. et milieu de vie naturel. Or, la résidence pour personnes âgées, n'étant pas un C.H.S.L.D., n'est-elle pas un milieu de vie naturel? Comment déterminer alors en quoi ce milieu de vie naturel est moins un domicile qu'un autre milieu de vie naturel? L'Agence de l'Estrie émet d'ailleurs comme l'un de ses principes directeurs que "(l)e milieu naturel est reconnu comme milieu de vie privilégié. Lorsque des services d'aide sont requis, on doit privilégier le recours à des ressources légères qui favorisent le maintien de la personne en perte d'autonomie liée au vieillissement dans son milieu de vie", sans cependant définir ce qu'elle entend par milieu de vie naturel) ; Régie Régionale de la Santé et des Services Sociaux de l'Estrie, Cadre de référence concernant les personnes âgées en perte d'autonomie demeurant dans des ressources d'habitation privées, Document de travail soumis à la consultation, Sherbrooke, 1999 (la question demeure troublante puisque, dans un document antérieur, la Régie Régionale de l'Estrie définissait le milieu de vie naturel comme un concept faisant "référence au fait de vivre au sein d'une communauté à laquelle la personne s'identifie et y obtient une possibilité d'exercer des rôles sociaux. Les résidences privées sont incluses dans la définition de milieu de vie naturel" [nos italiques]). 
des établissements, d'édicter une telle distinction dans l'accessibilité des services.

On pourrait cependant se questionner sur la constitutionnalité d'une telle mesure. Ne s'agirait-il pas d'une atteinte directe à l'article 15 de la Charte canadienne des droits et libertés ${ }^{79}$ qui stipule :

La loi ne fait acception de personne et s'applique également à tous, et tous ont droit à la même protection et au même bénéfice de la loi, indépendamment de toute discrimination, notamment des discriminations fondées sur la race, l'origine nationale ou ethnique, la couleur, la religion, le sexe, l'âge ou les déficiences mentales ou physiques $^{80}$.

Il s'agit d'une liste qui n'est pas exhaustive et donc, le lieu de résidence comme critère d'exclusion d'accès à un service offert habituellement par le C.L.S.C. serait source de discrimination contrevenant à la Charte canadienne ${ }^{81}$, pour autant qu'il rencontre l'examen du motif "analogue»82. "La portée de la protection contre

79. Law c. Canada (Ministère de l'Emploi et de l'Immigration) [1999] 1 R.C.S. 497 [Law] (la Cour suprême du Canada rappelle, notamment dans cet arrêt, la nécessité de fonder la constatation d'une discrimination en vertu de cette disposition sur trois éléments essentiels. Le premier étant de démontrer que la mesure contestée entraîne une différence de traitement par rapport à d'autres personnes. Nous explicitons plus loin les deux autres éléments qui doivent nécessairement être rencontrés).

80. Charte Canadienne des droits et libertés, art. 15(1), partie I de la Loi constitutionnelle de 1982, constituant l'annexe B de la Loi de 1982 sur le Canada (R-U), 1982, c.11 [Charte canadienne].

81. Ibid., art. 32 (1)b) (rappelons ici que la Charte canadienne s'applique «à la législature et au gouvernement de chaque province, pour tous les domaines relevant de cette législature». Il faudrait donc démontrer le fait direct du gouvernement provincial dans cette discrimination) ; Voir Patrick A. Molinari, "L'accès", supra note 29 à la p. 56.

82. Corbiere c. Canada (Ministre des Affaires indiennes et du Nord canadien) [1999] 2 R.C.S. 5203 [Corbiere] (un motif de distinction doit en effet rencontrer certains critères pour démontrer l'analogie avec un motif énuméré) ; Daniel Proulx, "Les droits à l'égalité revus et corrigés par la Cour suprême du Canada dans l'arrêt Law: un pas en avant ou un pas en ar- 
L'accès aux services d'aide à l'hygiène en résidence pour personnes âgées : $\quad$ (2007) 38 R.D.U.S. une atteinte aux principes d'égalité et de gratuité?

la discrimination de l'article 15 ne s'étend [donc] pas à toutes les distinctions arbitraires de l'État, mais uniquement à celles qui prennent appui ou ont un effet sur les motifs énumérés dans cette disposition ou analogues à ceux-ci» 83 . Peut-on considérer le lieu de résidence d'un individu comme étant un motif de distinction analogue, en l'espèce? La démonstration nous apparaît difficile...

Par ailleurs, la mesure gouvernementale doit "être réellement discriminatoire en ce qu'elle entre en conflit avec l'objet fondamental du paragraphe 15(1), c'est-à-dire la protection de la dignité humaine»84. Ainsi, l'arrêt $L a w^{85}$ fait état de quatre facteurs qui constituent les piliers du test de l'atteinte à la dignité. Ils sont : 1) La préexistence d'un désavantage, de stéréotypes, de préjugés ou d'une situation de vulnérabilité subie par le demandeur86; 2) La correspondance entre l'allégation de discrimination et la situation propre du demandeur ${ }^{87}$; 3) L'objet ou l'effet améliorateur de la mesure contestée à l'égard d'autres groupes défavorisés

rière?" (2001) 61 R.D.B. aux pp. 221-222 [Daniel Proulx, "Les droits à l'égalité"] (l'arrêt Corbiere a retenu deux critères-clé essentiels, "soit la nature "fondamentale» d'une caractéristique donnée pour une personne, c'est-à-dire l'importance de cette caractéristique pour l'identité personnelle, d'une part, et, d'autre part, le caractère "immuable dans les faits" (comme la race) ou "considéré immuable" d'une caractéristique parce qu'elle est hors du contrôle d'un individu ou parce que cela ne peut être changé qu'à un prix personnel inacceptable (comme la religion)) ; Corbiere, ibid. au para. 13 (Il s'agit donc de caractéristiques qu'il nous est impossible de changer ou encore, qu'il ne serait pas légitime que le gouvernement s'attende à ce que nous changions pour accéder à une égalité de traitement).

83. Daniel Proulx, "Les droits à l'égalité», Ibid. aux pp. 221-222.

84. David Robitaille, "Vous êtes victime de discrimination et vous souhaitez en faire la preuve? Bonne chance!» (2002) 62 R. du B. 319 [David Robitaille, "Vous êtes victime»].

85. Law, supra note 79.

86. Ibid. au para. 64 (il ne s'agit cependant pas d'une preuve indispensable à la reconnaissance d'une violation de la dignité).

87. David Robitaille, "Vous êtes victime", supra note 84 n.30 (ce critère sert à vérifier si la mesure contestée tient compte de la situation propre du demandeur. Ainsi, "(u)ne loi qui n'a pas pour objet ou effet d'accommoder un groupe d'individus peut en revanche correspondre à sa situation réelle dans la mesure où, d'un point de vue égalitaire, il n'a pas besoin de l'avantage duquel il est exclu' [nos italiques]). 
dans la société88; 4) La nature et l'étendue du droit ou de l'intérêt touché par la mesure contestée 89 .

Ce n'est donc qu'en franchissant chacune de ces étapes qu'une personne qui se croit lésée dans l'accès à un service habituellement offert par le C.L.S.C. pourrait obtenir reconnaissance d'une discrimination basée sur son lieu de domicile en vertu de l'article 15; il y a donc loin de la coupe aux lèvres ${ }^{90} .$.

88. Ibid. à la p.328 (ce critère sert à vérifier si la mesure contestée cherche à améliorer la situation d'un autre groupe défavorisé. Robitaille rappelle cependant que "les dispositions législatives qui visent une amélioration pour la population en général mais qui excluent des demandeurs historiquement défavorisés seront presque toujours taxées de discriminatoires", ce qui ne nous semble pas être le cas en l'espèce, les personnes âgées habitant en résidence privée pouvant difficilement être considérées comme faisant partie d'un groupe historiquement défavorisé).

89. Law, supra note 79 au para. 74 ; David Robitaille, "Vous êtes victime», supra note 84 à la p. 328 (Ce critère sert à évaluer l'importance économique, sociale et constitutionnelle du droit ou de l'intérêt auquel la mesure contestée porte atteinte et à vérifier si cette mesure entraîne l'exclusion d'une institution sociale fondamentale ou compromet la pleine appartenance à la société. Ainsi, la probabilité que la distinction soit discriminatoire au sens de l'article 15 de la Charte canadienne est proportionnelle à la gravité et à la délimitation de la conséquence pour le groupe touché).

90. Patrick A. Molinari, "L'accès", supra note 29 à la p. 56 (Cet auteur souligne qu'il n'est pas évident que la Charte canadienne trouve application. Il cite à ce sujet l'arrêt Stoffman c. Vancouver General Hospital [1990] 3 R.C.S. 483 où on a conclu au non assujettissement des hôpitaux à la Charte canadienne. L'arrêt Eldridge c. Colombie-Britannique nuance toutefois cette affirmation. En effet, une entité privée, en l'espèce un hôpital, serait assujettie à la Charte canadienne au même titre que le gouvernement lui-même s'il était prouvé qu'elle agissait en vertu de la mise en oeuvre d'une politique ou d'un programme gouvernemental déterminé. "Dans de telles circonstances, même si c'est un acteur privé qui exécute effectivement le programme, le gouvernement en conserve néanmoins la responsabilité. La justification de ce principe est facile à discerner. Tout comme il est interdit aux gouvernements de se soustraire à l'examen fondé sur la Charte en concluant des contrats commerciaux ou d'autres accords "privés", ils ne devraient pas être autorisés à échapper à leurs obligations constitutionnelles en déléguant la mise en ouvre de leurs politiques et programmes à des entités privées." Eldridge c. ColombieBritannique (Procureur général), 19973 R.C.S. 624.). 
L'accès aux services d'aide à l'hygiène en résidence pour personnes âgées : $\quad$ (2007) 38 R.D.U.S. une atteinte aux principes d'égalité et de gratuité?

Par ailleurs, on ne peut ici, selon nous, invoquer le droit à la vie et à la sécurité puisqu'il ne s'agit pas de soins médicaux ou de soins urgents et que par ailleurs, ceux-ci sont tout de même offerts, moyennant des coûts, nous en convenons ${ }^{91}$. En ce qui a trait à la Charte des droits et libertés de la personne ${ }^{92}$, il y a peu d'application dans la situation qui fait l'objet de notre analyse ${ }^{93}$, si ce n'est l'article 1, pour lequel les mêmes conclusions que celles relatives à l'article 7 de la Charte canadienne peuvent être tirées. À moins de considérer qu'une telle restriction d'accès aux soins pour les personnes âgées vivant en résidence privée ne constitue une forme d'exploitation telle que le prohibe l'article 48, ce qui ne nous paraît tout de même pas être le cas en l'espèce ${ }^{94}$ !

La conclusion qui doit être tirée ici est que, outre une invocation lointaine des Chartes, rien n'oblige juridiquement l'instance locale à fournir des services d'aide à l'hygiène en résidence pour personnes âgées.

91. La question pourrait tout de même se poser dans certains cas d'espèce. Par exemple, si la personne a peu ou pas de moyens financiers et si effectivement, ces soins lui étant ainsi inaccessibles, son intégrité ou sa sécurité en était menacée.

92. Charte des droits et libertés de la personne, L.R.Q., c. C-12 [Charte québécoise].

93. La discrimination sur la base du lieu de résidence ne peut être invoquée en vertu de l'article 10, s'agissant d'une liste définie de critères d'exclusion.

94. Charte québécoise, supra note 92, art. 48 ("Toute personne âgée ou toute personne handicapée a droit d'être protégée contre toute forme d'exploitation. Telle personne a aussi droit à la protection et à la sécurité que doivent lui apporter sa famille ou les personnes qui en tiennent lieu") ; Commission des droits de la personne c. Gagné [2003] R.J.Q. 647 (T.D.P.Q.) aux para. 82-84 (la notion d'exploitation doit être interprétée ici de façon large et ne se limite pas au cadre strictement économique. "L'exploitation comporte également une dimension d'ordre physique, psychologique, sociale ou morale. En fait, l'exploitation s'entend de toute situation de vulnérabilité et de dépendance d'une personne face à une autre qui, volontairement ou involontairement, en profite de manière abusive, et de la volonté de quelqu'un d'en profiter. L'exploitation s'entend, dans son sens vernaculaire, d'un profit abusif" [nos italiques]). 
Toutefois, sans explorer profondément cette voie, le cadre administratif dont se sont dotés le Ministère et les Agences laisse supposer que l'aide à l'hygiène constitue un service requis par l'état de santé de l'usager et que seul le fait qu'il soit assumé monétairement par l'usager lui-même justifie l'établissement de ne pas le dispenser. En d'autres termes, les politiques du Ministère et des Agences semblent convenir qu'il s'agit là d'un service requis auquel ils se soustraient pour l'unique motif que l'usager l'assume personnellement. À notre connaissance, un centre hospitalier ne se retire pas de ses obligations lorsqu'un usager a les moyens financiers d'assumer ses frais hospitaliers.

La seule formule législativement autorisée de formule de contribution des usagers est celle des articles 512 et 513 de la L.S.S.S.S. qui énoncent que :

512. Le gouvernement détermine, par règlement, la contribution qui peut être exigée des usagers qui sont hébergés dans une installation maintenue par un établissement public ou privé conventionné ou qui sont pris en charge par une ressource intermédiaire d'un établissement public ou par une ressource de type familial.

Ce règlement détermine également le montant d'allocation de dépenses personnelles qui doit être laissé mensuellement à cet usager.

513. Le montant de la contribution peut varier suivant les circonstances ou les besoins identifiés par règlement. Usagers visés.

La contribution est exigée par un établissement ou par le ministre. Les usagers eux-mêmes sont tenus de la verser; toutefois, dans le cas d'un usager mineur, la contribution peut être exigée de son père, de sa mère, de l'un et l'autre conjointement ou de toute autre personne déterminée par le règlement; dans le cas d'un usager marié ou uni civilement, la contribution peut être exigée de son conjoint et dans le cas d'un membre d'une communauté religieuse, la contribution peut être exigée de sa communauté95.

95. L.S.S.S.S., supra note 3, art. 512 et 513. 
Nous devons retenir trois choses du libellé de ces deux articles. Premièrement, la participation n'est autorisée que pour les services d'hébergement. Deuxièmement, seul le gouvernement détient le pouvoir d'instaurer une telle formule, excluant d'emblée un établissement tel un C.S.S.S. Finalement, la formule de participation ne peut être mise en place que par le biais d'un seul véhicule législatif, le règlement. Or, aucune de ces trois conditions n'est respectée, en l'espèce.

N'était-ce du flou des politiques du Ministère et des Agences et de la difficulté de qualifier l'aide à l'hygiène comme un service requis, il serait probablement possible de conclure que seul le gouvernement peut instaurer une formule de participation, qu'il doit le faire par voie de règlement et que celle-ci ne peut, à ce jour, porter uniquement que sur les coûts d'hébergement. Si l'on peut, à la rigueur, interpréter le maintien à domicile comme une forme d'hébergement, encore faut-il que ce soit le véritable titulaire du pouvoir qui l'exerce par voie réglementaire. Ce que l'on constate actuellement relève essentiellement de l'initiative des instances locales non détentrices d'un tel pouvoir et par voie décisionnelle quelquefois essentiellement clinique. Compte tenu que ces imprécisions dans les termes et les définitions rendent précaires des conclusions en droit, nous avons plutôt choisi d'explorer cet aspect au plan de l'éthique.

\section{III - Questionnement éthique et pistes de solution}

Le contexte législatif et réglementaire actuel crée, à notre avis, une situation d'iniquité en termes d'accessibilité et de gratuité pour les usagers qui nécessitent de l'aide à l'hygiène.

Il nous semble tout d'abord important de souligner que la Politique de soutien à domicile du M.S.S.S. détermine des critères de priorités dans l'obtention de ces services. Ceux-ci sont les besoins exprimés par l'usager et ses proches-aidants, l'urgence de la réponse aux besoins et la qualité du milieu de vie. On affirme qu' 
2007) 38 L'accès aux services d'aide à l'hygiène e

résidence pour personnes âgées :

une atteinte aux principes d'égalité et de gratuité?

(e)n aucun cas, des critères socioéconomiques ne sauraient constituer un motif de refus. Les services à domicile forment une composante des services de santé et des services sociaux et, à ce titre, ils sont accessibles à tous sans égard au revenu [nos italiques]. ${ }^{96}$

Or, dans les faits, la capacité de payer d'un individu ${ }^{97}$ fait souvent partie des critères de priorité cliniques dans l'accès aux services ${ }^{98}$.

Les personnes socioéconomiquement défavorisées sont habituellement desservies en priorité99. Mais qu'entend-on par le terme "personnes socioéconomiquement défavorisées"? Les personnes sous le seuil de la pauvreté? En Estrie, environ $18 \%$ des personnes âgées en ménage privé vivent sous le seuil de faible revenu, soit $24 \%$ des femmes et $11 \%$ des hommes et près de $44 \%$ des personnes âgées qui vivent seules sont sous le seuil de faible revenu, soit $48 \%$ des femmes et $34 \%$ des hommes ${ }^{100}$. Une personne habitant en H.L.M. ayant les mêmes revenus qu'une personne habitant en résidence privée pour personnes âgées ne dispose-t-elle pas de plus de revenus disponibles que la deuxième? Cette dernière n'est-elle pas ainsi plus défavorisée "socioéconomiquement parlant" que la première? Des ambigüités subsistent donc en regard de la priorité d'accès aux services à domicile basée sur le revenu.

Outre l'iniquité au sein de la clientèle desservie par la mission C.L.S.C., nous pouvons questionner l'équité sur une base plus large, soit au sein de l'ensemble de la clientèle desservie par

96. Ministère de la Santé et des Services sociaux, Pour faire les bons choix, supra note 68 à la p. 19.

97. Ou encore son éligibilité à un remboursement par une assurance privée des frais encourus pour un service donné.

98. Nous reviendrons un peu plus loin sur les choix de l'établissement dans l'accès aux services et la réalité des listes d'attente.

99. Il s'agissait d'ailleurs d'un critère de priorité définit antérieurement par le M.S.S.S. à l'intérieur de son Cadre de référence sur les services à domicile de première ligne.

100. Agence de développement de réseaux locaux de services de santé et de services sociaux Estrie, Programme-services, supra note 47 à la p. 23. 
le C.S.S.S. Attardons-nous sur les différents types de services dispensés directement par l'instance locale, à savoir les services hospitaliers électifs, les services d'hébergement public et les services à domicile. Il est opportun ici de faire un parallèle entre ceux-ci en regard des stratégies utilisées dans la gestion des ressources, limitées il va sans dire. Ceci permettra de bien faire ressortir le questionnement éthique relatif à la question qui nous occupe101.

Nous savons que les services cliniques fournis en vertu de la mission de centre hospitalier se doivent d'être gratuits. Il est approprié de penser que les services périphériques ${ }^{102}$ sont inclus dans cette obligation puisqu'il s'agit de services nécessaires à la dispensation des services cliniques ${ }^{103}$. Le contrôle des coûts s'établit donc en fonction d'une barrière externe, à savoir l'accès même aux services cliniques hospitaliers ${ }^{104}$.

En ce qui a trait aux services d'hébergement public, le législateur a opté pour un partage des coûts financiers des services périphériques fournis en vertu de la mission C.H.S.L.D. Les usagers hébergés dans le réseau public doivent ainsi débourser une part des coûts de leur hébergement ${ }^{105}$. Les coûts sont donc régulés par une barrière interne, à savoir une limite à la gratuité106.

101. Le schéma 1 illustre notre propos (Annexe 1).

102. On parle ici de tout l'aspect de l'hébergement et des soins découlant de cet hébergement, à savoir les repas, l'entretien ménager, l'aide à l'hygiène, notamment.

103. À titre d'exemple, une chirurgie de la cataracte ne pourrait être considérée comme un service clinique nécessitant des services périphériques hospitaliers, s'agissant d'une chirurgie d'un jour. Nous excluons bien sûr les circonstances d'une complication nécessitant une hospitalisation.

104. Québec, Ministère de la santé et des services sociaux, "Accès aux services médicaux spécialisés", en ligne: M.S.S.S. <http://wpp01.msss. gouv.qc.ca/appl/g74web/> (est-il besoin de rappeler les nombreuses listes d'attente pour les services médicaux spécialisés dans les centres hospitaliers du Québec? Le M.S.S.S. a rendu accessible des données sur l'état de la situation sur son site Internet. Le sujet fait également la manchette de façon régulière dans les différents médias d'information).

105. L.S.S.S.S., supra note 3, art. 512 ; Règlement d'application de la Loi sur les services de santé et les services sociaux, R.R.Q., 1981, c. S-5, r.1 art. 358-377.1 (cependant, tel que souligné précédemment, il y a un plafond 
Les services à domicile, quant à eux, occupent une position mitoyenne. Le contexte tel que nous l'avons exposé démontre un recours à une barrière interne par le biais d'une tarification "cachée" pour certains usagers. Mais il existe également, comme nous le démontrerons plus loin, une limite à l'accessibilité par le biais des listes d'attente (barrière externe). Les services à domicile useraient donc d'une barrière hybride.

Cet état de fait semble découler non pas d'une réflexion appuyée sur des assises claires mais paraît plutôt émerger d'un tiraillement clinico-administratif issu de l'augmentation des besoins et de la limitation des ressources. Dans un effort d'optimisation des budgets disponibles, on assiste donc à des initiatives locales basées sur des critères décisionnels variés, pour ne pas dire aléa-

à une telle contribution et la personne est assurée de conserver une part de son revenu mensuel pour d'autres dépenses. Pour toute modalité relative à la tarification d'un adulte hébergé dans le réseau public voir le Règlement d'application de la Loi sur les services de santé et les services sociaux).

106. Agence de la santé et des services sociaux de l'Estrie, Rapport annuel de gestion 2005-2006, Sherbrooke, adopté le 25 octobre 2006 à la p. 35 (on peut peut-être s'étonner que les listes d'attente du réseau d'hébergement public ne fassent pas partie de la limite à l'accessibilité dans notre analyse. Or, il convient de rappeler que dans les faits, un usager nécessitant un hébergement et étant "en attente" de cet hébergement, est souvent pris en charge par le milieu hospitalier en occupant un lit de courte durée ou un lit dans un service spécialisé, par exemple les unités de réadaptation fonctionnelle intensive. En effet, le rapport annuel de gestion de l'Agence de l'Estrie fait état que "(l)es délais pour la clientèle en attente dans les services de la courte durée vers l'hébergement en centre d'hébergement de soins de longue durée se sont grandement améliorés passant de 43,84 jours en 2003 à 9,5 jours en 2005 pour le Centre hospitalier universitaire de Sherbrooke, de 46,5 jours à 11 jours pour l'Unité de réadaptation fonctionnelle intensive et de 17,62 à 10 jours pour l'Unité de courte durée gériatrique") ; Règlement sur la contribution des usagers des établissements de santé et de services sociaux, D.22-93, (1993) 125 G.O. II, 657, art. 1(2) (d'ailleurs, cette réalité est reconnue de façon réglementaire puisqu'une contribution financière de la personne est exigée à compter du quarante-cinquième jour d'hospitalisation, à moins que le médecin ne détermine que des soins actifs ne soient toujours requis). 
L'accès aux services d'aide à l'hygiène en résidence pour personnes âgées : $\quad$ (2007) 38 R.D.U.S. une atteinte aux principes d'égalité et de gratuité?

toires, de nature clinique ${ }^{107}$, juridique 108 et éthique ${ }^{109}$ afin de limiter les coûts"10. Le M.S.S.S. lui-même fait le constat qu' "(o)n observe certaines formes d'iniquité en ce qui concerne l'accès à l'évaluation et aux services ainsi que la détermination du caractère prioritaire ou urgent d'un besoin" [nos italiques].111

Compte tenu de l'inadéquation entre besoins identifiés et ressources pour y répondre, les listes d'attente pour les différents services à domicile dispensés par la mission C.L.S.C. sont généralisées. Le M.S.S.S. reconnaît que "(l)es délais d'attente ne sont pas toujours précisés ou ne respectent pas toujours les priorités d'évaluation annoncées. Il en va de même pour la gestion des listes d'attente qui n'existe pas ou n'est pas normalisée" [nos italiques] ${ }^{112}$. Nous pourrions même aller plus loin : dans l'état actuel des choses, les priorités dans l'accès aux services et l'attente en découlant se traduit dans les faits par de l'exclusion pure et simple $^{113}$ ! Le service, théoriquement accessible, ne l'est pas dans les faits, puisque la personne se trouve sur une liste d'attente pour des mois, parfois des années ${ }^{114}$.

107. On réfère ici bien sûr au projet clinique en lui-même et aux priorités ciblées en termes de besoins populationnels.

108. On réfère ici à la L.S.S.S.S. et tous les règlements en découlant.

109. On réfère ici aux choix organisationnels en termes de priorité dans l'accès aux services par la clientèle.

110. Le schéma 2 illustre la situation (Annexe 2).

111. Ministère de la santé et des services sociaux, Chez soi, supra note 46 à la p. 13.

112. Ibid.

113. Ministère de la Santé et des Services sociaux, Pour faire les bons choix, supra note 68 à la p. 19 (si par exemple, la personne a la capacité de payer et paie effectivement pour un service requis, le degré d'urgence n'est plus le même. Cette demande devient ainsi moins "prioritaire" qu'une autre. Pratique qui va à l'encontre de la position du M.S.S.S. qui dit qu' "(e)n outre, lorsqu'une personne ou ses proches trouvent une solution temporaire de dépannage parce qu'ils doivent subir un délai, ils ne doivent pas être pénalisés dans l'ordre de priorité établi parce qu'ils ont eu recours à cette solution").

114. Il n'est pas rare d'ailleurs que l'incapacité temporaire de l'usager se résorbe ou encore, que l'usager décède, avant même d'avoir pu bénéficier des soins requis! 
2007) 38 Laccès aux services d'aide à l'hygiène e résidence pour personnes âgées:

une atteinte aux principes d'égalité et de gratuité?

Il y a donc lieu, à notre avis, de se questionner sur cette réalité. Un soin requis de l'usager, à savoir l'aide à l'hygiène, est gratuit ou non, accessible ou non, selon la mission du C.S.S.S. à l'intérieur de laquelle il est dispensé. Loin de nous l'idée de plaider pour une uniformisation des critères d'une mission à l'autre! Mais il nous semblerait minimal qu'un raisonnement rationnel clair détermine les choix du législateur.

En effet, les services périphériques à domicile tels les soins d'aide à l'hygiène ne peuvent-ils être considérés comme des services professionnels au sens de la Politique du M.S.S.S., s'ils sont nécessaires à la dispensation des services cliniques ${ }^{115}$ ? Comment concilier les contraintes budgétaires, les besoins des usagers ${ }^{116}$ et les questionnements dégagés plus hauts? Deux solutions nous viennent spontanément.

La première implique un nivellement des services offerts par la mission C.L.S.C. du C.S.S.S. afin que la palette de ces services soit la même pour tous. À l'instar des services d'hébergement public, pourquoi ne pas imposer des frais à tous les usagers qui nécessitent de l'aide à l'hygiène, quel que soit leur domicile? ${ }^{117}$ On contourne une discrimination dans l'accès basée uniquement sur le lieu de résidence et on apporte également de l'argent supplémentaire au système, ce qui répond à l'impératif financier. Et fina-

115. Soulignons, sur ce point, l'ambigüité dont le M.S.S.S. fait preuve à notre avis. Plutôt que de parler de "services cliniques", on parle de "services professionnels". Ceux-ci sont pourtant dispensés dans le cadre du projet clinique du R.L.S. Qu'entend-on par services "professionnels»? Parle-t-on des services dispensés uniquement par des professionnels au sens du Code des professions? Tel semble être le cas; or, ne devrait-on pas faire la différence entre un "service professionnel" et un "service dispensé par un professionnel»? Il nous apparaît clairement que l'aide à l'hygiène ne peut qu'être un service professionnel, en raison de sa nature même.

116. Ministère de la santé et des services sociaux, Chez soi, supra note 46 à la p. 13 (d'autant que "(l)'évaluation des besoins est souvent effectuée en fonction de l'offre de services disponibles plutôt que sur la base des besoins de la personne»)!

117. Il va de soi qu'une telle option ne pourrait être réalisée que par le gouvernement et par voie réglementaire. Voir, à cet effet, notre conclusion de la section précédente. 
L'accès aux services d'aide à l'hygiène en résidence pour personnes âgées : $\quad$ (2007) 38 R.D.U.S. une atteinte aux principes d'égalité et de gratuité?

lement, il nous apparaît que cette solution est équitable pour tous et assure une répartition des frais à l'ensemble des citoyens consommateurs. Mais au nom de cette équité, peut-on imposer des frais à des usagers qui ont payé et paient toujours largement leur part? Il y a là un questionnement essentiel face à l'équité à tout prix.

La deuxième solution envisagée se veut un financement des services dans les résidences privées par le secteur public. Tout comme pour la mission C.H.S.G.S. du C.S.S.S., les soins d'aide à l'hygiène nécessaires à la prestation des services cliniques seraient fournis gratuitement à l'usager. Ceci pourrait se faire par les préposés de l'établissement ou par les ressources humaines de la résidence, au choix de l'usager. Dans ce cas, le secteur public, via une allocation financière à la résidence, assumerait les coûts ${ }^{118}$. Cette solution a l'avantage d'être équitable pour tous les usagers. Cependant, on peut se questionner sur la capacité réelle de l'Agence de s'assurer de la qualité des services dispensés par les préposés de la résidence privée. Et cette solution implique évidemment des dépenses supplémentaires pour le secteur public, qui, est-il besoin de le rappeler, affecte déjà une large part des recettes fiscales de l'État québécois au système de santé!

Chacune de ces solutions comporte son lot d'avantages et d'inconvénients. Bien d'autres auraient avantage à être explorées dans le futur.

118. L.S.S.S.S., supra note 3, art. 454 (d'ailleurs, comme nous l'avons précédemment souligné, cet article le permettrait. En effet, il stipule : "Afin de permettre à des personnes en perte d'autonomie de recevoir différents services de santé ou services sociaux, l'agence peut attribuer à une résidence privée d'hébergement ou à un établissement privé non conventionné qui exploite un centre d'hébergement et de soins de longue durée une allocation financière pouvant varier suivant la nature des services offerts". Cependant l'article 454 ne s'appliquant qu'aux établissements au sens de la loi, il ne trouve pas application auprès de ressources d'hébergement qui ne sont pas des établissements, même s'ils sont agréés. L'extension de cet article s'avère donc essentielle). 
(2007) 38 R.D.U.S. $\begin{gathered}\text { L'accès aux services d'aide à l'hygiène en } \\ \text { résidence pour personnes âgées : }\end{gathered}$

une atteinte aux principes d'égalité et de gratuité?

\section{Conclusion}

L'ensemble de notre réflexion nous a permis, entre autres, de faire ressortir les ambigüités relatives à la notion de domicile et les iniquités dans l'accès aux services d'aide à l'hygiène offerts par les C.S.S.S. Le législateur aurait à intervenir en la matière mais il serait fort étonnant qu'il procède à court terme, à moins de pressions sociales intenses. Quel avantage y aurait-il? Légiférer implique nécessairement un choix, un choix quant aux répercussions financières importantes, soit pour l'État, soit pour l'usager. La pression sur le système de santé va en augmentant avec le vieillissement de la population, particulièrement le groupe des très âgés. Le M.S.S.S. préférera sans nul doute laisser les initiatives locales, la libre-concurrence et les lois du marché régir l'état actuel des choses.

Les recours pour l'usager qui se croit lésé sont minimes. La Loi canadienne sur la santé ne s'applique pas aux services à domicile, tel que nous l'avons déjà déterminé. La L.S.S.S.S., pour sa part, prévoit des procédures de plaintes; cependant, comme il s'agit de choix liés à l'organisation des services et pouvant donc faire l'objet de règlements par le conseil d'administration de l'établissement, ce recours demeure limité. À moins d'incidents ouvrant un recours civil, la légalité même d'un tel règlement pourrait peut-être être contestée en vertu des Chartes, tel que nous l'avons déjà soulevé.

Nous voyons que le mythe populaire répandu que tous les services de santé et tous les services sociaux sont accessibles à tous, universels et gratuits de surcroît, prend racine dans une interprétation large de la Loi canadienne. Nous savons maintenant que la portée de celle-ci est limitée et que le droit aux services exigibles en vertu de la loi québécoise n'est pas absolu et est même tributaire des choix faits par les établissements eux-mêmes!

Quoique, nous l'avons constaté, l'inaction de l'État puisse constituer une charge inéquitable à l'endroit d'un groupe particulier, il convient de souligner qu'elle s'inscrit dans une réforme axée 
L'accès aux services d'aide à l'hygiène en résidence pour personnes âgées : $\quad$ (2007) 38 R.D.U.S. une atteinte aux principes d'égalité et de gratuité?

sur la première ligne qui n'en finit pas d'aboutir. Cette inaction relèverait donc du projet en cours de réalisation et sur lequel l'État compte. De cette réforme axée sur le citoyen, il ne faudrait toutefois pas ne retenir que le concept de citoyen payeur et ainsi, laisser au citoyen âgé la charge de payer seul la restructuration des services de santé et des services sociaux.

«(...) par la manière dont une société se comporte avec ses vieillards, elle dévoile sans équivoque la vérité - soigneusement masquée - de ses principes et de ses fins»119

119. Simone de Beauvoir, La vieillesse, essai, Paris, Gallimard, 1970 à la p. 96. 
L'accès aux services d'aide à l'hygiène en

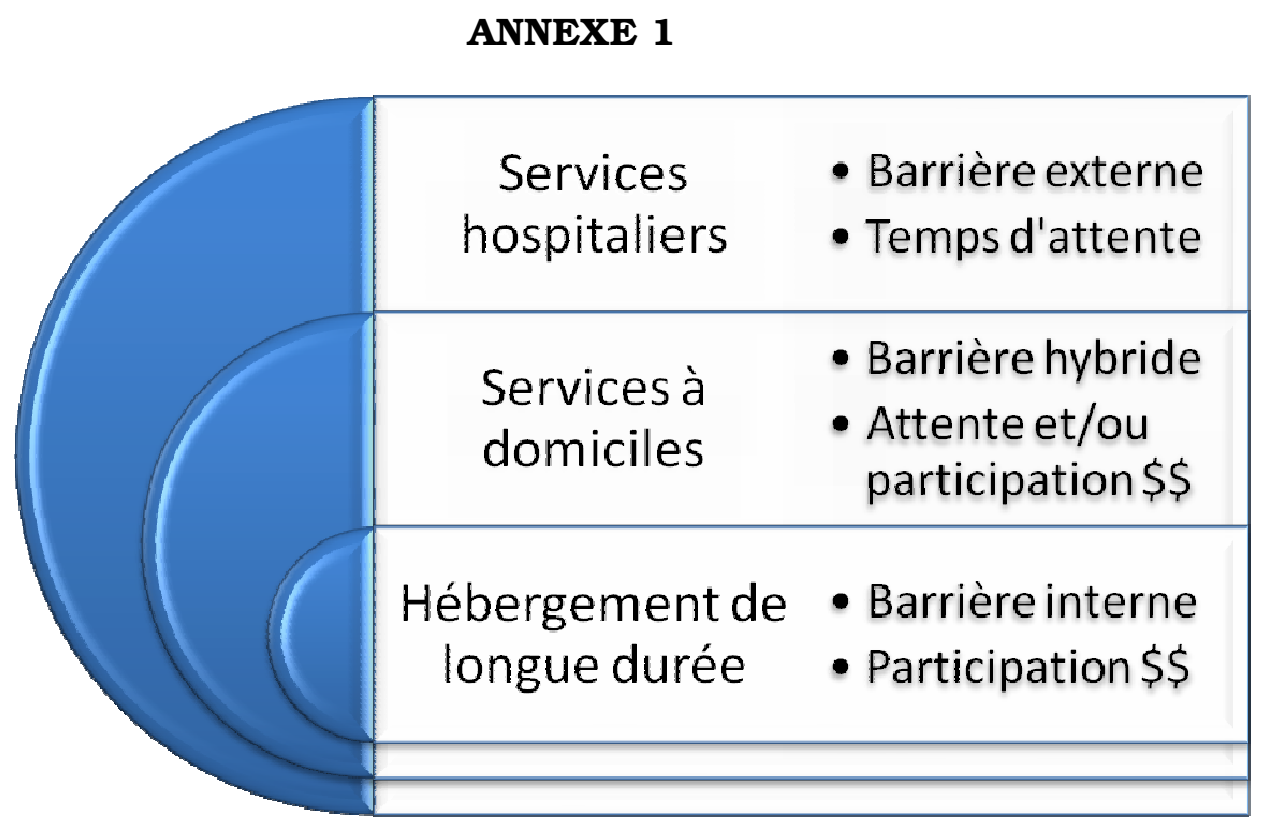

Schéma 1

\section{Stratégies de gestion des ressources limitées} selon le type de service.

Notez que si les temps d'attente sont juridiquement autorisés lorsque la gestion de l'établissement est adéquate, la participation financière de

l'usager doit être autorisée législativement. Actuellement, seuls l'hébergement et certains services cliniques ciblés peuvent faire l'objet d'une participation financière de l'usager. 


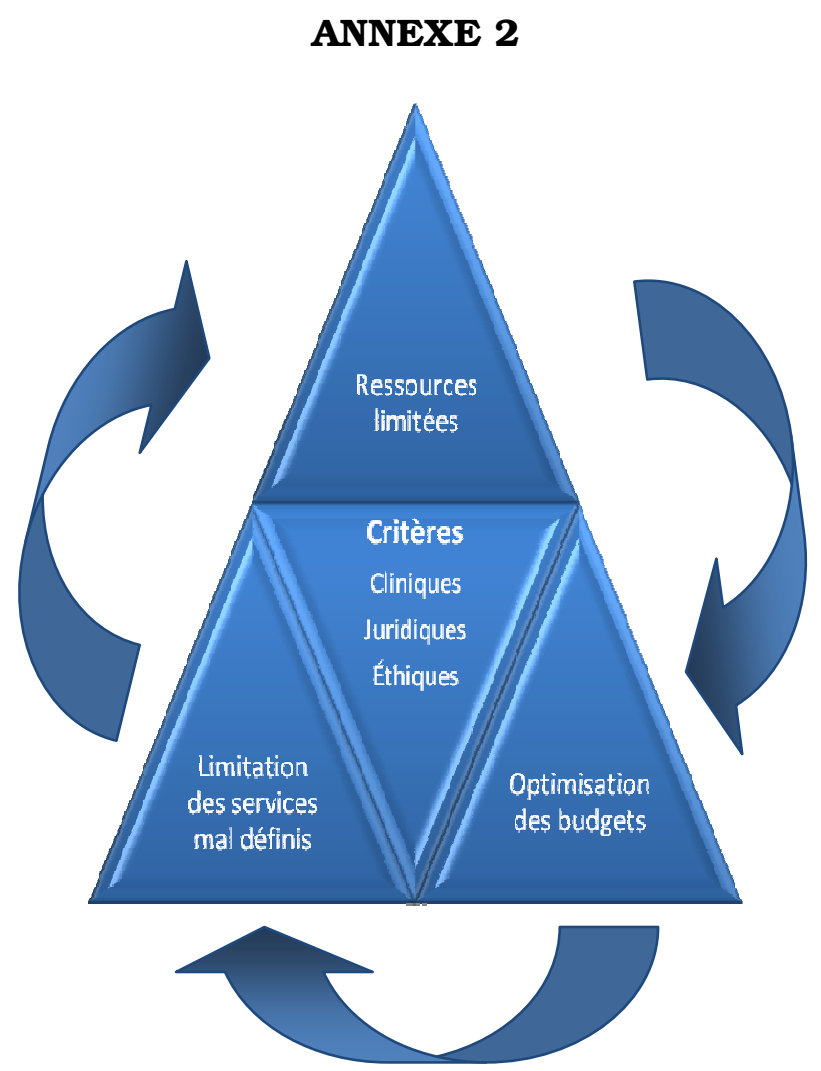

Schéma 2

Impact aléatoire des ressources limitées sur les services mal définis. 